Article

\title{
Thermal and Electromagnetic Combined Optimization Design of Dry Type Air Core Reactor
}

\author{
Fating Yuan ${ }^{1}$, Zhao Yuan ${ }^{1, *}$, Lixue Chen ${ }^{1}$, Yong Wang ${ }^{2}$, Junxiang Liu ${ }^{2}$, Junjia He ${ }^{1}$ \\ and Yuan Pan ${ }^{1}$ \\ 1 State Key Laboratory of Advanced Electromagnetic Engineering and Technology, \\ Huazhong University of Science and Technology, Wuhan 430074, China; yuanfatinghss@163.com (F.Y.); \\ chenlixue@hust.edu.cn (L.C.); h-junjia@vip.sina.com (J.H.); panyuan@mail.hust.edu.cn (Y.P.) \\ 2 Guangzhou Power Supply Co., Ltd., Guangzhou 510620, China; wangy@guangzhou.csg.cn (Y.W.); \\ jxliu_2006@126.com (J.L.) \\ * Correspondence:yuanzhao8507@163.com; Tel.: +86-027-8754-2474
}

Received: 28 September 2017; Accepted: 17 November 2017; Published: 1 December 2017

\begin{abstract}
In this paper, taking the minimum metal conductor usage of an air core reactor as optimization goal, the influence of air ducts width and encapsulation number on thermal efficiency and electromagnetic efficiency are analyzed. Combined with the equation constraint conditions for maximum temperature rise conservation, inductance conservation and structure function of reactor considering the change of air ducts width and encapsulation number, the thermal and electromagnetic combined optimization curves are formed, and design results are achieved based on the initial design parameters. Meanwhile, the temperature field simulation model of the reactor is established and the results verify the correctness of the optimization method. According to the design results, the proportionality factor of metal conductor usage is only 0.61 compared with the initial design parameters of the reactor, which shows that the proposed comprehensive optimization method can significantly reduce conductor usage, improving the metal conductor utilization ratio.
\end{abstract}

Keywords: air duct width; encapsulation number; temperature rise conservation; inductance conservation; temperature field; utilization ratio of metal conductors

\section{Introduction}

Dry-type air core reactors are now being widely used in power systems [1,2]. The design optimization of a reactor mainly focuses on the contradiction between the key properties (inductance, current carrying ability) and cost of the metal conductor materials (copper, aluminum). Thus, optimization methods to improve the utilization ratio of metal conductor are needed. Currently, the optimization theory and method in this field mainly include electromagnetic optimization and thermal optimization.

Regarding electromagnetic optimization, in [3], the optimal shape proportion for thick wall coils (also called "Huke curve") is given. It describes the percentage of metal conductor usage over the minimum when the shape proportion deviates from the optimal value. In [4], the magnetic particle layer is added outside of a conventional aluminum core to increase the permeability of the coils. However, the thermal conditions are not considered in [3,4]. Considering thermal optimization, in [5], the equal current density method, combined with the average temperature rise, is used to realize the same average temperature rise of encapsulation; in [6,7], the equal temperature rise and the equal potential drop of resistance method is used in the design of the reactor, which can achieve the same temperature rise of the inner encapsulation and the minimal overall loss of the reactor, however, the electromagnetic conditions are neglected in [5-7]. 
In [8-11], the genetic algorithm, improved genetic algorithm, and particle swarm optimization algorithm are applied in the design of the reactor; In [12,13], the finite element method is used to improve the utilization ratio of metal conductor, nevertheless, a large amount of computation and long optimization times are needed in order to obtain the optimal parameters of the reactor. Therefore, the optimization methods mentioned above cannot be widely used.

In $[14,15]$, the thermal and electromagnetic optimization are combined in the design of the reactor, however, the change of the air duct width and encapsulation number are not simultaneously considered, which directly affects the electromagnetic and thermal characteristics of the reactor, and eventually lead to the results that the design parameters not being optimal. Thus, the air duct width and encapsulation number should be taken into account. Currently, the optimization methods concerning the air duct width and encapsulation number mainly include the following: in [16], the empirical value of the air duct width can be obtained based on the heat and heat dissipation conditions of the encapsulations; in [17], the traversal algorithm is used to optimize the encapsulation number and other parameters, but the long optimization time is the main drawback. Therefore, these methods cannot be used effectively in the design of reactors.

In this paper, a comprehensive optimization method is proposed considering the change of the air duct width and encapsulation number, to realize the minimum metal conductor usage of the reactor. Combined with the equality constraint conditions for maximum temperature rise conservation, inductance conservation and structure function of the reactor, the optimization curves and results are obtained based on the initial design parameters of the reactor, and the correctness of the optimization method is verified by the temperature field simulation results. At the same time, the design results show that the optimization method can obviously reduce metal conductor usage, which is of great guiding significance to the design of the reactor.

\section{Methods}

The optimization goal is to realize the minimum metal conductor usage of the reactor. The goal function can be expressed as:

$$
\operatorname{minMass}=\pi \rho_{1} \sum_{i=1}^{m} W_{i} D_{i} S_{i}
$$

where Mass is the total mass of metal conductor, $\rho_{1}$ is the mass density of metal conductor, $W_{i}, D_{i}$ and $S_{i}$ are the turn number, diameter, and single turn conductor cross-sections of encapsulation $i$, and $m$ is the encapsulation number.

In order to achieve the minimum metal conductor usage, the influences of the air duct width and the encapsulation number on the thermal and electromagnetic efficiency are taken into account, meanwhile, a comprehensive optimization method is proposed considering the change of the air duct width and the encapsulation number.

\subsection{Thermal and Electromagnetic Optimization}

The equal height and heat flux design method is used in the design of the reactor, which can guarantee that the height, heat flux, radial width, and current density of each encapsulation are basically equal, which provides the precondition for thermal and electromagnetic efficiency optimization of the reactor. Table 1 shows the initial design parameters based on equal height and the heat flux design method (the metal conductor material is aluminum), which is selected as the optimization object to analyze the thermal and electromagnetic efficiency considering the change of the air duct width and the encapsulation number. 
Table 1. The initial design parameters of air core reactor.

\begin{tabular}{cc}
\hline Reactor & Parameters \\
\hline Rated inductance $(\mathrm{mH})$ & 20.9 \\
Rated current $(\mathrm{A})$ & 875.5 \\
Height of the reactor $(\mathrm{m})$ & 1.3 \\
Inside diameter $(\mathrm{m})$ & 1.6 \\
Outside diameter $(\mathrm{m})$ & 2.4 \\
Width of air ducts $(\mathrm{m})$ & 0.025 \\
Average turn number & 122.5 \\
Number of encapsulation & 12 \\
Maximum temperature rise $\left({ }^{\circ} \mathrm{C}\right)$ & 48 \\
\hline
\end{tabular}

\subsubsection{The Thermal Efficiency Optimization}

The thermal efficiency optimization goal is to achieve the minimum metal conductor usage, and the constraint condition is the maximum temperature rise conservation. Considering the heat and heat dissipation conditions of the inner encapsulations are basically the same, based on the equal height and heat flux design method, the encapsulation-air ducts unit, which is comprised of two encapsulations and an air duct, can be taken as the research object to compute the maximum temperature rise of the coils. For a large-type air core reactor, the diameter of the encapsulation is larger than the air duct width, so the unit can be considered equivalent to a vertical tube tank model, as shown in Figure 1.

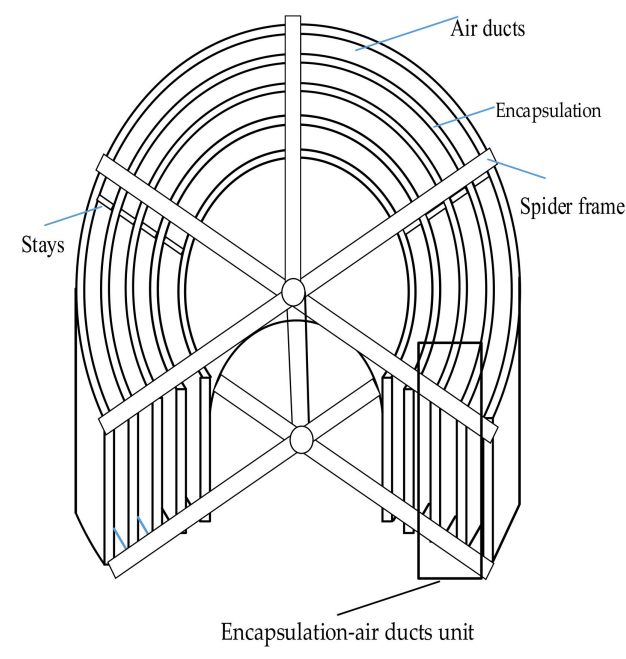

(a)

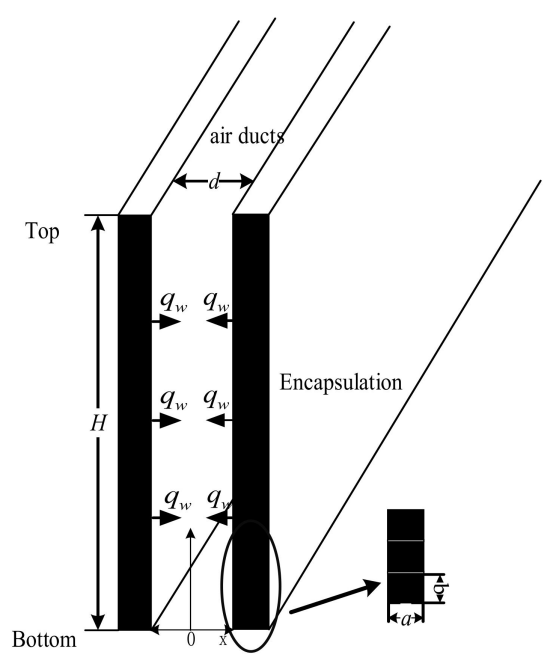

(b)

Figure 1. The basic structure of the reactor and equivalent encapsulation-air ducts unit. (a) The basic structure of air core reactor. (b) Encapsulation-air ducts unit.

Assume the variable in the initial design is $x_{0}$, which is selected as the normalized standard, the corresponding variable in a new design is $x$. Then, the proportionality factor is defined as $k_{x}=x / x_{0}$.

The heat flux conducted from the encapsulation surface to the air ducts is evenly distributed based on the equal height and heat flux design method. According to the heat transfer process in a vertical tube tank, the maximum temperature rise of encapsulation, which is located nearly at the top of the encapsulation, and can be equal to the sum of the average fluid temperature rise and fluid-solid temperature difference $[15,18]$, as shown in Equation (2), and the accuracy has been validated by experimental results:

$$
T_{\text {max }}=\frac{2 q_{w} H}{d \bar{u} \rho C_{p}}+\frac{2 q_{w} d}{\lambda N u}
$$


where, in the right-hand side of the equation, the first term is the average fluid temperature rise, and the second term is the fluid-solid temperature difference. $T_{\max }$ is the maximum temperature rise of encapsulation, $q_{w}$ is the heat flux conducted from the encapsulation surface to air ducts, $H$ is the height of the encapsulation, $d$ is the width of the air ducts, $\bar{u}$ is the average velocity of the fluid, $\rho$ is the fluid density, and $C_{p}$ is the specific heat at constant pressure. $\lambda$ is the heat conductivity coefficient of the fluid, and $N u$ is the Nusselt number in the air ducts.

The heat flux $q_{w}$ in Equation (2) can be written as:

$$
q_{w}=\frac{k_{c} I^{2}}{2 \gamma a b^{2}}
$$

where $k_{c}$ is the ratio of total losses to resistance losses, $I$ is the encapsulation current, $\gamma$ is the conductivity of metal conductor, $a$ is the radial width of encapsulation, and $b$ is the single turn axial height of the encapsulation.

According to the Equations (2) and (3), the thermal efficiency optimization can be realized by adjusting the ratio of the fluid-solid temperature difference and average fluid temperature rise.

(1) Considering the change of the air duct width

The thermal efficiency of encapsulation-air ducts unit can be adjusted by changing the variable parameters $a, b, d$ and $H$. Assume the conductor section size in the initial design parameters are $a$ and $b$, respectively, the values become $a^{\prime}$ and $b^{\prime}$ after adjustment. Thus, $k_{a}=a^{\prime} / a, k_{b}=b^{\prime} / b$.

The encapsulation current is decided by the total current and current distribution principles of the reactor, so it is regarded as a constant in the adjustment process of the encapsulation-air ducts unit, and the conductivity of the metal conductor remains unchanged. As a result, the proportionality factor of heat flux can be written as Equation (4):

$$
k_{q w 1}=\frac{1}{k_{a} k_{b}^{2}}
$$

Combining Equations (2) and (4), the proportionality factor of the maximum temperature rise considering the change of the air duct width can be written as Equation (5):

$$
k_{T \max }=\frac{1}{k_{a} k_{b}^{2}} \frac{\lambda N u k_{N u} H k_{\bar{W}} k_{b}+\left(k_{d} d\right)^{2} \bar{u} k_{\bar{u}} \rho C_{p}}{\left(\lambda N u H+d^{2} \bar{u} \rho C_{p}\right) k_{N u} k_{\bar{u}} k_{d}}
$$

where $k_{\bar{W}}, k_{N u}, k_{\bar{u}}$, and $k_{d}$ are the proportionality factors of the average turn number, Nusselt number, the fluid average velocity, and the air duct width, respectively.

In encapsulation-air ducts units, combined with the quality equation, momentum equation, and boundary conditions, the axial velocity of the fluid along the radial direction in the air ducts can be calculated according to Equation (6) [19,20]:

$$
u=\frac{g \beta d^{2}\left(T-T_{\infty}\right)}{8 v}\left[1-\left(\frac{x}{d / 2}\right)^{2}\right]
$$

where $g$ is the acceleration due to gravity, $\beta$ is the thermal expansion coefficient, $T$ is the temperature of encapsulation surface, $T_{\infty}$ is the temperature of fluid, $v$ is the kinematic viscosity of fluid, and $x$ is the radial length from the middle of the air ducts to encapsulation surface.

The mass flow rate of fluid $\bar{M}$ in the air ducts is:

$$
\bar{M}=\frac{\rho g \beta d^{3}\left(T-T_{\infty}\right)}{24 v}
$$


Suppose the heat flux conducted from the encapsulation surface to the air ducts is completely used to heat the fluid in the air ducts; the equation can be written as:

$$
c_{p} \bar{M}\left(T-T_{\infty}\right)=2 q_{w} H
$$

Combined with Equations (6)-(8), the average velocity of fluid in air ducts can be written as:

$$
\bar{u}=\sqrt{g \beta d q_{w} H /\left(12 \mu C_{p}\right)}
$$

where $\mu$ is the dynamic viscosity of the fluid, combined with the average fluid velocity and the experimental correlation equation in the vertical ducts, the Nusselt number $N u$ in Equation (5) can be obtained, and the detailed computation process can be found in Appendix A.

The proportionality factor of metal conductor usage considering the change of the air duct width can be written as:

$$
k_{\text {Mass }}=k_{a} k_{b} k_{\bar{W}} k_{\text {Dav }}
$$

The electromagnetic character is ignored, supposing the average turn number and diameter remain unchanged in the adjustment process of the encapsulation-air ducts unit, namely $k_{\bar{W}}=1$, $k_{D a v}=1$. Therefore, the proportionality factor of metal conductor usage can also be written as Equation (11):

$$
k_{\text {Mass }}=k_{a} k_{b}
$$

From Equations (2)-(9), the parameters $k_{a}, k_{u}$ and $k_{N u}$ can be replaced by $k_{b}$ and $k_{d}$, respectively, combined with the initial design parameters of the reactor and fluid properties in the air ducts, it can be derived that the proportionality factor of metal conductor usage, $k_{\text {Mass }}$, is a function of $k_{b}$ and $k_{d}$, as shown in Equation (12):

$$
k_{\text {Mass }}=\frac{1}{k_{b}} \frac{\lambda N u k_{N u} H k_{\bar{W}} k_{b}+\left(k_{d} d\right)^{2} \bar{u} k_{\bar{u}} \rho C_{p}}{\left(\lambda N u H+d^{2} \bar{u} \rho C_{p}\right) k_{N u} k_{\bar{u}} k_{d}}=f\left(k_{b}, k_{d}\right)
$$

For a given range of variables parameter $k_{b}$ and $k_{d}$, the optimization curve can be plotted which describes the thermal efficiency of the unit considering the change of air duct width, as shown in Figure 2.

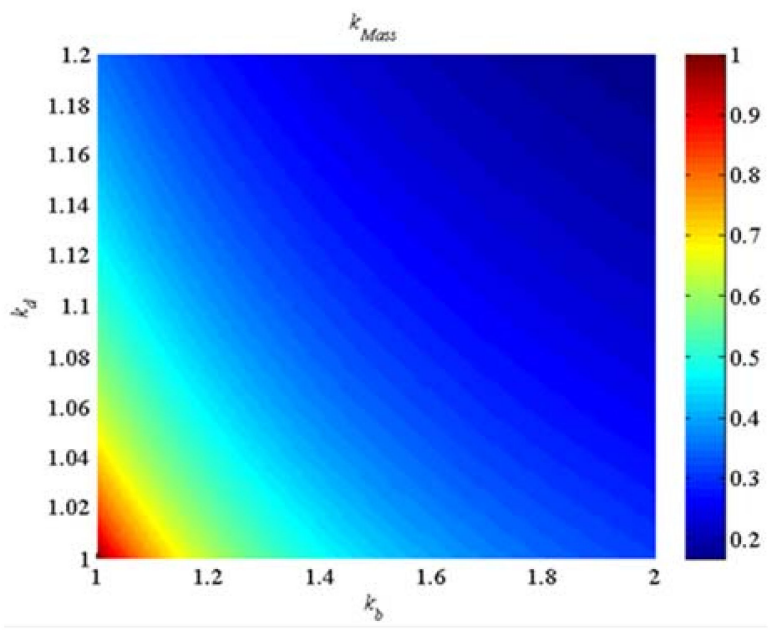

Figure 2. Thermal efficiency optimization curve considering the change of the air duct width. 
As Figure 2 shows, the proportionality factor of metal conductor usage, $k_{\text {Mass }}$ is gradually decreased with the increase of $k_{b}$ and $k_{d}$. Thus, it can be concluded that the thermal efficiency can be improved by increasing the encapsulation height and the air duct width.

According to Equation (5), the thermal efficiency optimization curves are plotted with the different initial parameters of the unit, the encapsulation number is taken as a constant, and the variable parameters are the air duct width and encapsulation height, as shown in Figure 3. In Figure 3, when the air duct width remains unchanged, the further optimization effect is obviously reduced with the increase of the encapsulation height; when the encapsulation height remains unchanged, the further optimization effect is gradually increased with the increase of the air duct width, and the effect is limited. The main reason is that the increase of the encapsulation height leads to the ratio (the fluid-solid temperature difference and average fluid temperature rise) decreasing; however, the increase of the air duct width leads to the ratio increasing.

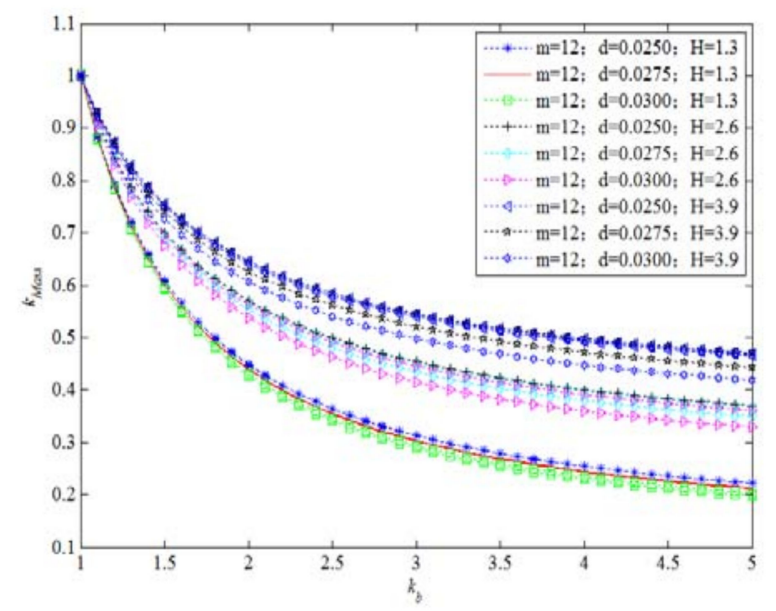

Figure 3. Thermal efficiency optimization curves with the different initial parameters.

(2) Considering the change of encapsulation number

According to the heat transfer process of encapsulation-air ducts unit, the proportionality factor of maximum temperature rise considering the change of encapsulation number can be written as Equation (13):

$$
k_{T \max }=k_{q w 2} \frac{\lambda N u k_{N u} H k_{\bar{W}} k_{b}+d^{2} \bar{u} k_{\bar{u}} \rho C_{p}}{\left(\lambda N u H+d^{2} \bar{u} \rho C_{p}\right) k_{N u} k_{\bar{u}}}
$$

The proportionality factor of heat flux can be written as:

$$
k_{q w 2}=\frac{1}{k_{a} k_{b}^{2} k_{m}^{2}}
$$

The proportionality factor of metal conductor usage is:

$$
k_{\text {Mass }}=k_{a} k_{b} k_{\bar{W}} k_{\text {Dav }} k_{m}
$$

Similarly, ignoring the change of the average turn number and the diameter of the encapsulation, namely, $k_{\bar{W}}=1, k_{D a v}=1$, the proportionality factor of metal conductor usage can be written as Equation (16):

$$
k_{\text {Mass }}=k_{a} k_{b} k_{m}
$$

From Equations (9), (13) and (14) and Appendix A, the parameters $k_{a}, k_{\bar{u}}$, and $k_{N u}$ can be replaced by $k_{b}$ and $k_{m}$, combined with the initial design parameters of the reactor and fluid properties in the air 
ducts, it can be derived that the proportionality factor of metal conductor usage, $k_{\text {Mass }}$, is a function of $k_{b}$ and $k_{m}$, as shown in Equation (17):

$$
k_{\text {Mass }}=\frac{1}{k_{b} k_{m}} \frac{\lambda N u k_{N u} H k_{\bar{W}} k_{b}+d^{2} \bar{u} k_{\bar{u}} \rho C_{p}}{\left(\lambda N u H+d^{2} \bar{u} \rho C_{p}\right) k_{N u} k_{\bar{u}}}=f\left(k_{b}, k_{m}\right)
$$

For a given range of variable parameters $k_{b}$ and $k_{m}$, the optimization curve is plotted which describes the thermal efficiency of the unit considering the change of the encapsulation number, as shown in Figure 4 . As Figure 4 shows, the proportionality factor of metal conductor usage, $k_{\text {Mass }}$, is gradually decreased with the increase of $k_{b}$ and $k_{m}$. Thus, the thermal efficiency can be improved by increasing the encapsulation number.

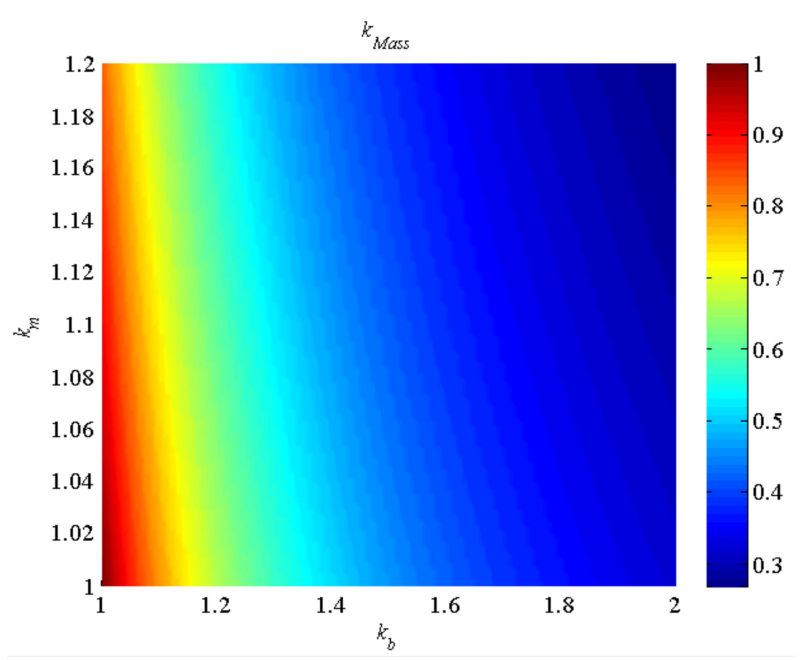

Figure 4. Thermal efficiency optimization curve considering the change of encapsulation number.

According to Equation (13), the thermal efficiency optimization curves are plotted with the different initial parameters of the unit. As shown in Figure 5, the air duct width is taken as a constant, and the variable parameters are the encapsulation number and encapsulation height.

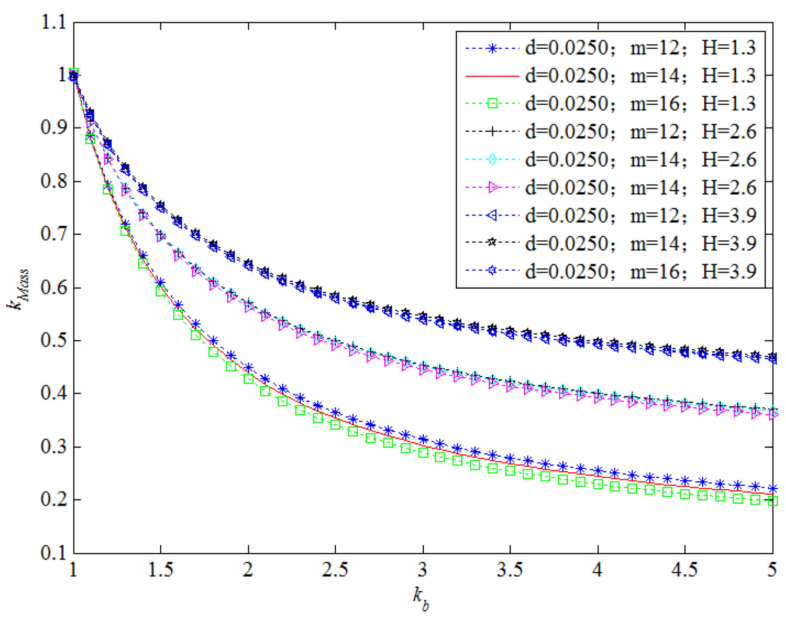

Figure 5. Thermal efficiency optimization curves with the different initial parameters.

In Figure 5, when the encapsulation number remains unchanged, the further optimization effect is obviously reduced with the increase of the encapsulation height; when the encapsulation height 
remains unchanged, the further optimization effect is gradually increased with the increase of the encapsulation number, and the effect is limited. The main reason is that the ratio (the fluid-solid temperature difference and the average fluid temperature rise) is decreased with the increase of the encapsulation height, but it is increased with the increase of the encapsulation number.

\subsubsection{The Electromagnetic Efficiency Optimization}

The optimization goal of electromagnetic efficiency is to achieve the minimum metal conductor usage, and the constraint condition is the inductance conservation. The essence of both the air core reactor with multiple coaxial encapsulations and thick wall coils are same from the aspect of electromagnetic efficiency realization. Therefore, the actual air core reactor can be equivalent to a single thick wall coil with the same proportion, as shown in Figure 6.

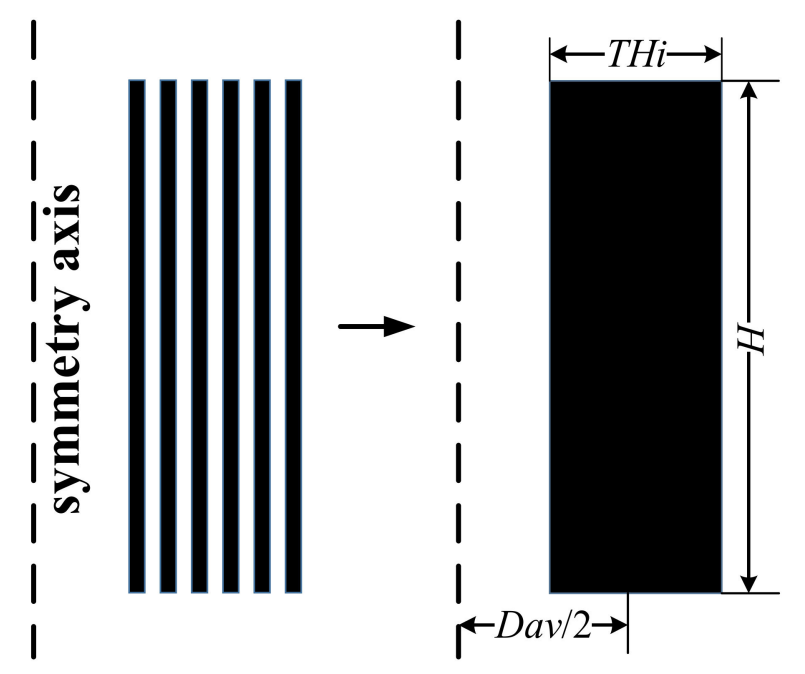

Figure 6. The equivalent aspect ratio of the reactor.

In Figure 6, H, THi, and Dav are the height, thickness and average diameter of the thick wall coils. The geometry factors, $\alpha$ and $\beta$, are defined as [15]:

$$
\left\{\begin{array}{l}
\alpha=H / D a v \\
\beta=T H i / D a v
\end{array}\right.
$$

According to the equivalent thick wall coils, Equation (19) is selected as the constraint condition for inductance conservation, and the accuracy is widely accepted [21]:

$$
L=\frac{7.85 \bar{W}^{2} \mathrm{Dav}^{2}}{3 \mathrm{Dav}+9 \mathrm{H}+10 \mathrm{THi}} \times 10^{-6}
$$

where $L$ is the value of the inductance.

In order to build the relationship between the inductance and air duct width, encapsulation number, the structural function of the reactor is established, as shown in Equation (20):

$$
\left\{\begin{array}{l}
\text { THi }=m(a+d) \\
H=\bar{W} \cdot b
\end{array}\right.
$$

where, $m$ is the encapsulation number. 
(1) Considering the change of air duct width

The thermal character is ignored, assuming the current density and average diameter of the encapsulation remains unchanged, namely $k_{a} k_{b}=1, k_{D a v}=1$. Therefore, according to Equation (10), the proportionality factor of metal conductor usage, $k_{\text {Mass }}$, can also be written as Equation (21):

$$
k_{\text {Mass }}=k_{\bar{W}}
$$

From Equations (19) and (20), $k_{\bar{W}}$ can be replaced by $k_{b}$ and $k_{d}$, combined with the initial design parameters of the reactor, which can derive that the proportionality factor of metal conductor usage, $k_{\text {Mass }}$, is a function of $k_{b}$ and $k_{d}$, as shown in Equation (22):

$$
k_{\text {Mass }}=\sqrt{\frac{3 D a v+9 H k_{b} k_{\bar{W}}+10 T H i\left(\left(a / k_{b}+k_{d} d\right) /(a+d)\right)}{3 D a v+9 H+10 T H i}}=f\left(k_{b}, k_{d}\right)
$$

For a given range of variable parameters $k_{b}$ and $k_{d}$, the optimization curve can be plotted according to the Equation (22), which describes the electromagnetic efficiency of the reactor considering the change of the air duct width, as shown in Figure 7.

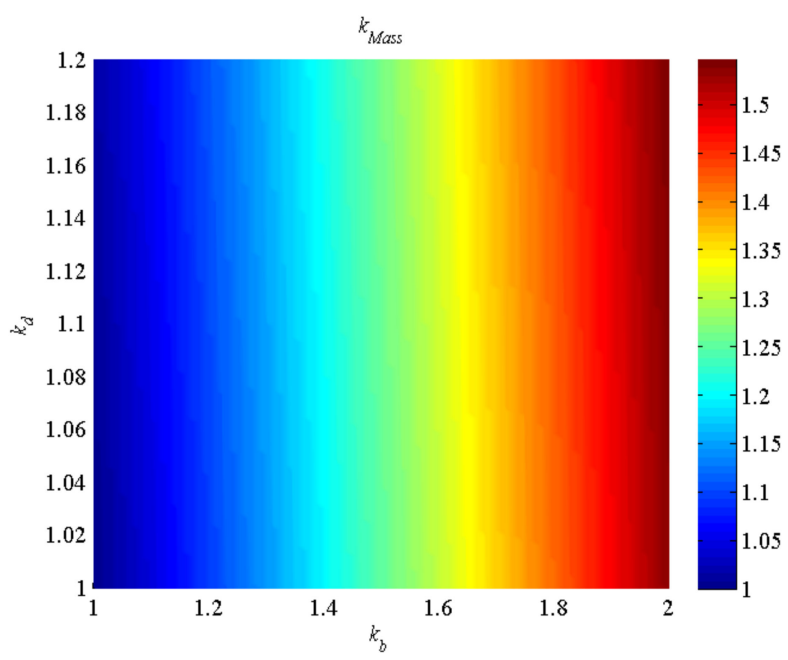

Figure 7. Electromagnetic efficiency optimization curve considering the change of the air duct width.

As shown in Figure 7, the proportionality factor of metal conductor usage, $k_{\text {Mass }}$ is gradually increased with the increase of $k_{b}$, and the change of the air duct width, $k_{d}$ has less of an effect on it.

(2) Considering the change of encapsulation number

Similarly, ignoring the change of current density and the average diameter of the encapsulation, assume the value of $k_{a} k_{b} k_{m}=1, k_{D a v}=1$, combined with Equations (18)-(20), the proportionality factor of metal conductor usage considering the change of the encapsulation number can be written as:

$$
k_{\text {Mass }}=\sqrt{\frac{3 D a v+9 H k_{b} k_{\bar{W}}+10 T H i k m\left(\left(d+a / k_{b} k_{m}\right) /(a+d)\right)}{3 D a v+9 H+10 T H i}}=f\left(k_{b}, k_{m}\right)
$$

For a given range of variables parameter $k_{b}$ and $k_{m}$, the optimization curve can be plotted according to Equation (23), which describes the electromagnetic efficiency of the reactor considering the change of the encapsulation number, as shown in Figure 8. 


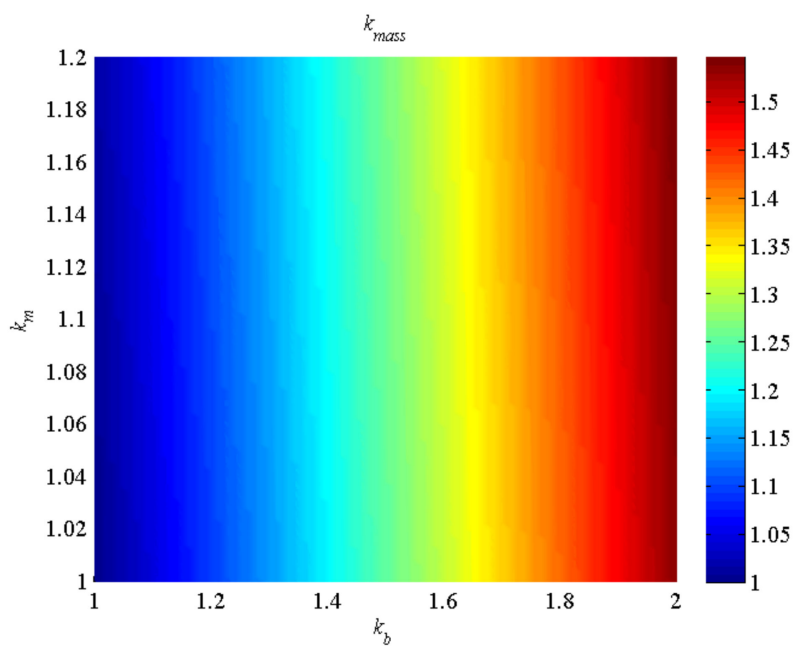

Figure 8. Electromagnetic efficiency optimization curve considering the change of the encapsulation number.

In Figure 8, the proportionality factor of metal conductor usage, $k_{\text {Mass }}$ is gradually increased with the increase of $k_{b}$, and the change of encapsulation number, $k_{m}$ has less of an effect on it. At the same time, the change trend of curve is basically the same as the curve in Figure 7.

\subsection{The Comprehensive Optimization Method}

According to the above analysis regarding thermal and electromagnetic efficiency optimization, it can be concluded that the increase of the air duct width, encapsulation number, and encapsulation height can improve the thermal efficiency of the unit, but the electromagnetic efficiency of the coils is decreased. Thus, a comprehensive optimization method is proposed considering the change of the air duct width and encapsulation number, to realize the metal conductor usage minimum.

\subsubsection{Equality Constraint Conditions}

The comprehensive optimization method needs to meet inductance conservation, maximum temperature rise conservation, and the structural function of the reactor.

(1) Maximum temperature rise conservation

According to the heat transfer process of the encapsulation-air ducts unit, the proportionality factor of the maximum temperature rise considering the change of the air duct width and encapsulation number can be written as Equation (24), and the heat flux is shown in Equation (25):

$$
\begin{gathered}
k_{T \max }=k_{q w} \frac{\lambda N u k_{N u} H k_{\bar{W}} k_{b}+k_{d}^{2} d^{2} \bar{u} k_{\bar{u}} \rho C_{p}}{\left(\lambda N u H+d^{2} \bar{u} \rho C_{p}\right) k_{N u} k_{\bar{u}} k_{d}} \\
k_{q w}=\frac{1}{k_{a} k_{b}^{2} k_{m}^{2}}
\end{gathered}
$$

(2) Inductance conservation

According to Equation (19), the constraint condition for inductance conservation can also be written as:

$$
k_{\bar{W}}=\left(\frac{3+9 \alpha^{\prime}+10 \beta^{\prime}}{3+9 \alpha+10 \beta} / k_{D a v}\right)^{0.5}
$$


(3) Structural function

The change of the air duct width and encapsulation number will inevitably affect the overall structure of the reactor, combined with Equations (18) and (20), the proportionality factor of the radial width and single turn axial height of encapsulation can be written as:

$$
\begin{gathered}
k_{a}=\frac{2 \beta^{\prime} k_{D a v}}{\beta k_{m}}-k_{d} \\
k_{b}=\frac{k_{D a v} \alpha^{\prime} / \alpha}{k_{\bar{W}}}
\end{gathered}
$$

\subsubsection{The Realization of the Optimization Method}

According to Equations (24)-(28), the constraint conditions considering the change of the air duct width and encapsulation number can be redefined as:

$$
\begin{aligned}
& k_{a}=\frac{1}{k_{b}^{2} k_{m}^{2}} \frac{\lambda N u k_{N u} H k_{\bar{W}} k_{b}+\left(k_{d} d\right)^{2} \bar{u} k_{\bar{u}} \rho C_{p}}{\left(\lambda N u H+d^{2} u \rho C_{p}\right) k_{N u} k_{\bar{u}} k_{d}} \\
& k_{\bar{W}}=\left(\frac{3+9 \alpha^{\prime}+10 \beta^{\prime}}{3+9 \alpha+10 \beta} / k_{D a v}\right) .5 \\
& k_{D a v}=\frac{\left(k_{a}+k_{d}\right) \beta k_{m}}{2 \beta^{\prime}} \\
& k_{b}=\frac{k_{D a v} \alpha^{\prime} / \alpha}{k_{\bar{W}}}
\end{aligned}
$$

where $\alpha$ and $\beta$ are the initial geometry factors, and $\alpha^{\prime}$ and $\beta^{\prime}$ are the optimized geometry factors.

Thus, the realization of comprehensive optimization method becomes the analysis of metal conductor usage. According to Equation (29), the parameters of $k_{a}, k_{b}, k_{\bar{W}}$, and $k_{\text {Dav }}$ can be replaced by $\alpha^{\prime}, \beta^{\prime}, k_{d}$ and $k_{m}$, and it can be derived that the proportionality factor of metal conductor usage, $k_{\text {Mass }}$ is a function of the four independent variables $\alpha^{\prime}, \beta^{\prime}, k_{d}$ and $k_{m}$, as shown in Equation (30):

$$
k_{\text {Mass }}=f\left(\alpha^{\prime}, \beta^{\prime}, k_{d}, k_{m}\right)
$$

In [15], the air duct width and encapsulation number are taken as constants, the values of $\alpha^{\prime}$ ranges from 0.5 to 1.6 , and $\beta^{\prime}$ ranges from 0.2 to 1 , according to the curves, the minimum value of $k_{\text {Mass }}$ can be obtained when $\alpha^{\prime}$ is ca. 1.5 and $\beta^{\prime}$ is ca. 0.2 , and the correctness has been validated by the design results. Thus, the comprehensive optimization method adopts $\alpha^{\prime}=1.5$ and $\beta^{\prime}=0.2$ in Equation (30), and the proportionality factor of metal conductor usage can be rewritten as:

$$
k_{\text {Mass }}=f\left(k_{d}, k_{m}\right)
$$

From Equation (31), $k_{\text {Mass }}$ is decided by two independent variables $k_{d}$ and $k_{m}$, thus, for a given range of $k_{d}$ and $k_{m}$, the optimization curve considering the change of the air duct width and encapsulation number can be plotted.

\subsubsection{The Overall Optimization Process}

In order to realize the metal conductor usage minimum of the reactor considering the change of the air duct width and encapsulation number, three steps are indispensable in the optimization process:

Step 1: Taking the initial parameters of the reactor based on equal height and heat flux design methods as the optimization object, combined with inductance conservation, the maximum temperature rise conservation and structure function of the reactor, and the optimization curve considering the change of the air duct width and encapsulation number can be achieved.

Step 2: According to the optimization curve in Step 1, the optimal value of $k_{d}$ and $k_{m}$ can be obtained when $k_{\text {Mass }}$ reaches the minimum value, combined with the initial parameters, the equal height, and heat flux design method of the reactor, the optimization design results can be achieved. 
Step 3: According to the optimization design results in Step 2, a fluid-thermal-coupled finite element model is established to calculate the maximum temperature rise of the reactor, taking the temperature field simulation results as the evaluation criterion of the optimization method.

The overall optimization design process of the reactor is shown in Figure 9.

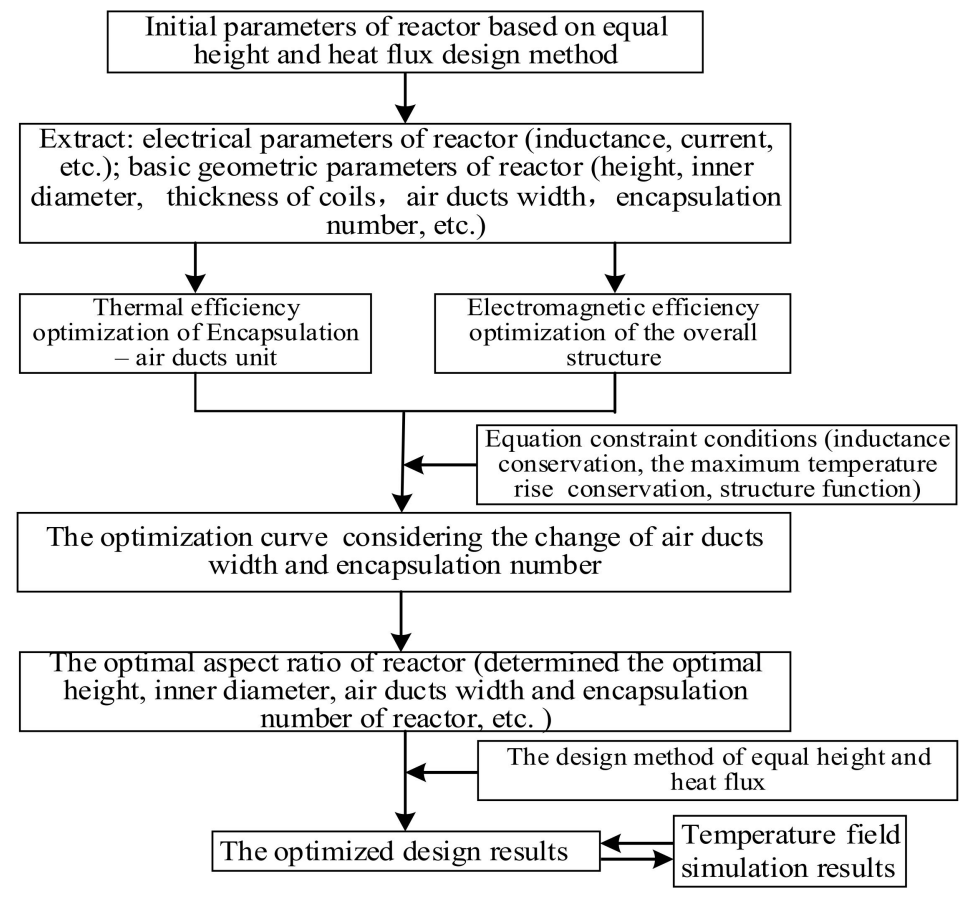

Figure 9. The overall optimization design process of the reactor.

\section{Results}

A certain type of air core reactor is given in order to prove the utility of optimization theory and methods, and the parameters of the reactor in Table 1 are selected as the optimization object.

\subsection{Optimization Curves}

\subsubsection{Considering Only the Change of Air Duct Width}

In the natural air cooling condition, when the encapsulation number remains constant, combined with the fluid properties parameters in air ducts and Equation (30), setting $\beta^{\prime}=0.2$, the optimization curve considering the change of the air duct width can be calculated and plotted, as shown in Figure 10. The minimum value of $k_{\text {Mass }}$ is 0.73 when $\alpha^{\prime}$ is nearly 1.5 and $k_{d}$ is nearly 1.1. 


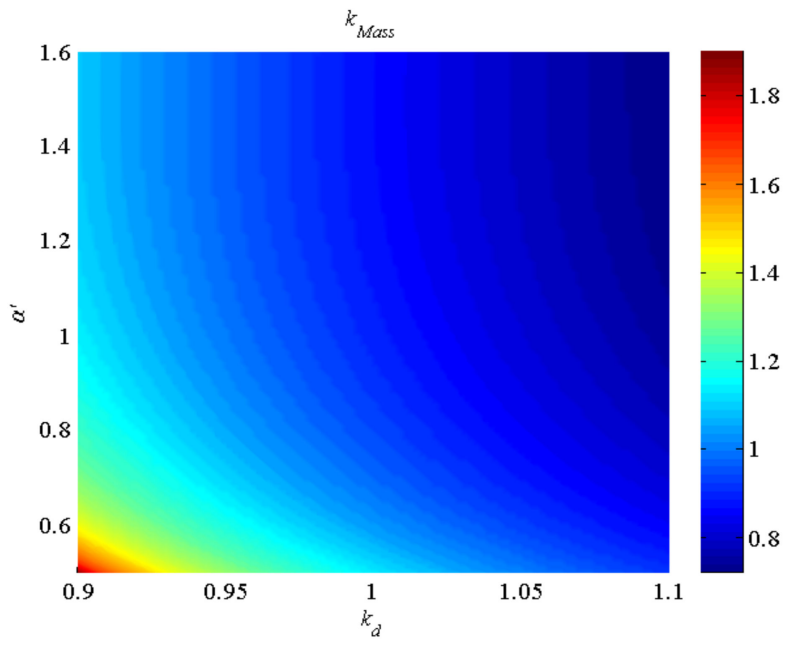

Figure 10. The thermal and electromagnetic combined optimization curve considering the change of the air duct width.

\subsubsection{Considering Both the Change of Air Duct Width and Encapsulation Number}

Similarly, combined with the fluid properties parameters in air ducts and Equation (30), setting the $\alpha^{\prime}=1.5$ and $\beta^{\prime}=0.2$, the optimization curve considering the change of the air duct width and encapsulation number can be calculated and plotted, as shown in Figure 11. The minimum value of $k_{\text {Mass }}$ is 0.6 when the value of $k_{d}$ is nearly 1.1 and $k_{m}$ is nearly 1.16 . Thus, compared with the initial parameters of the reactor, the comprehensive optimization method can obviously reduce metal conductor usage.

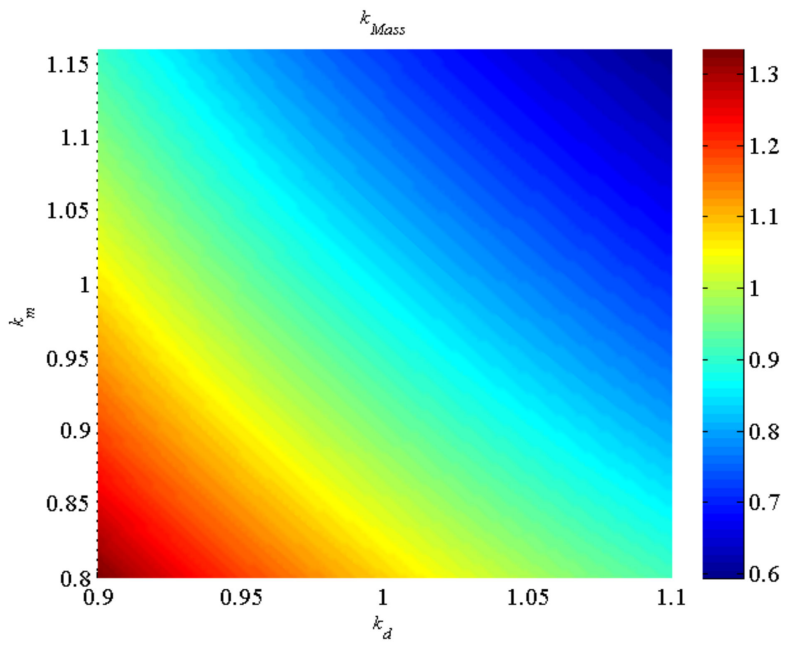

Figure 11. The thermal and electromagnetic combined optimization curve considering the change of air duct width and encapsulation number.

In the comprehensive optimization method, the change of the air duct width and encapsulation number inevitably lead to the change of other parameters. Figures 12 and 13 are the $k_{R}$ and $k_{H}$ curves considering the change of the air duct width and encapsulation number, and $R$ is the direct current (DC) resistance of the reactor.

In Figure 12, the DC resistance is gradually increased with the increase of the air duct width and encapsulation number, and the trends are reversed compared with Figure 11, and the maximum value of $k_{R}$ is 2.38 when $k_{d}$ is nearly 1.1 and $k_{m}$ is nearly 1.16 . 
In Figure 13, it can be seen that the encapsulation height is gradually increasing with the increase of the encapsulation number; the maximum value of $k_{H}$ is 1.91 when $k_{d}$ is nearly 1.1 and $k_{m}$ is nearly 1.16 .

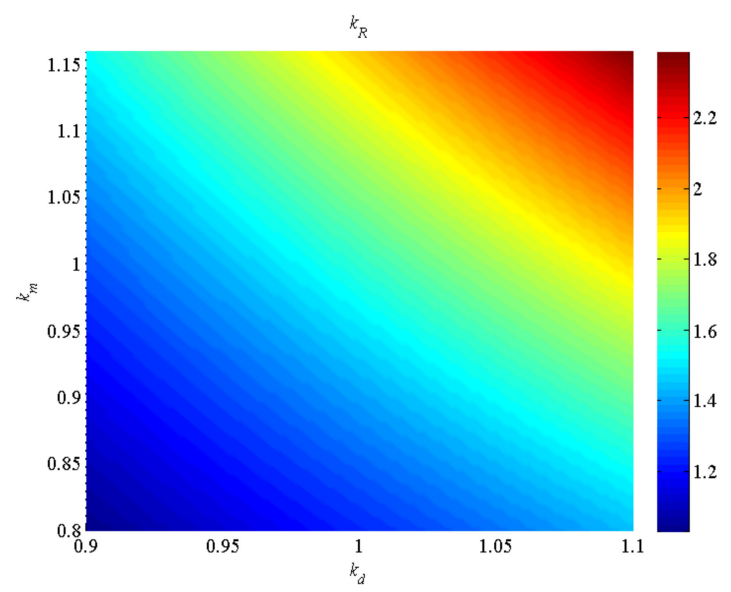

Figure 12. The $k_{R}$ curve considering the change of air duct width and encapsulation number.

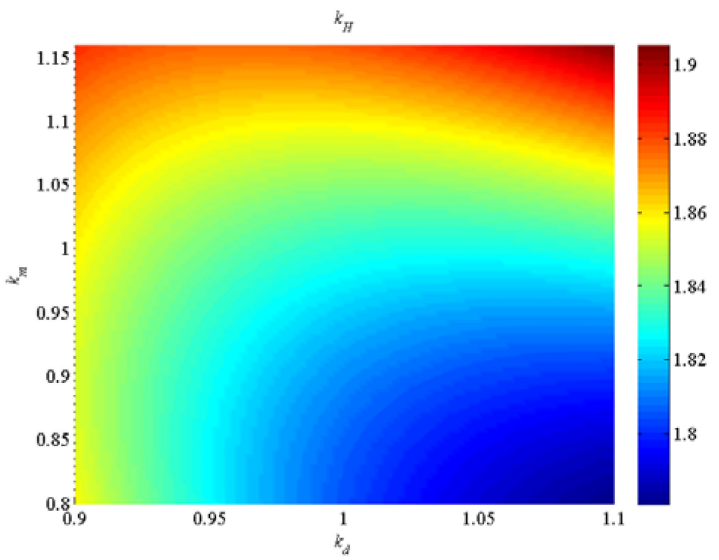

Figure 13. The $k_{H}$ curve considering the change of air duct width and encapsulation number.

\subsection{Design Results}

The design results are shown in Table 2, Method 1 is the equal height and heat flux design method, Method 2 is the design method only considering the change of the air duct width; and Method 3 is the comprehensive optimization method considering the change of the air duct width and encapsulation number.

Table 2. The results of three design methods.

\begin{tabular}{cccc}
\hline Method & $\mathbf{1}$ & $\mathbf{2}$ & $\mathbf{3}$ \\
\hline$H(\mathrm{~m})$ & 1.3 & 2.58 & 2.48 \\
THi $(\mathrm{m})$ & 0.390 & 0.397 & 0.42 \\
$\operatorname{Dav}(\mathrm{m})$ & 1.99 & 1.72 & 1.77 \\
$H / D a v$ & 0.65 & 1.5 & 1.4 \\
THi $/$ Dav & 0.2 & 0.231 & 0.24 \\
$\bar{W}$ & 122.5 & 175 & 169 \\
$d(\mathrm{~m})$ & 0.025 & 0.0275 & 0.0275 \\
$m$ & 12 & 12 & 14 \\
Mass $(\mathrm{t})$ & 1.62 & 1.18 & 0.99 \\
$K_{\text {Mass }}$ & 1 & 0.73 & 0.61 \\
\hline
\end{tabular}


In Table 2, the proportionality factor of metal conductor usage is 0.73 in Method 2, and is only 0.61 in Method 3, which shows that the comprehensive optimization method can obviously reduce metal conductor usage. Meanwhile, when adopting the comprehensive optimization method, the influence on thermal efficiency and electromagnetic efficiency of the reactor are analyzed.

Thermal efficiency: according to the analysis of thermal efficiency in encapsulation-air ducts unit, the proportionality factor of metal conductor usage gradually decreased with the increase of the air duct width, encapsulation number, and height. In Method 3, the value of $k_{H}$ is $1.91, k_{d}$ is 1.1 , and $k_{m}$ is 1.16 , compared with the initial parameters of the reactor; thus, the thermal efficiency is improved with the comprehensive optimization method.

Electromagnetic efficiency: according to the analysis of electromagnetic efficiency of the reactor, the proportionality factor of metal conductor usage is gradually increased with the increase of the encapsulation height. In Method 3, the value of $k_{H}$ is 1.91, and the average turn number ranges from 122.5 to 169 ; thus, the electromagnetic efficiency is reduced with the comprehensive optimization method.

\subsection{Simulation Verification}

Inductance and maximum temperature rise are the main parameters of the reactor. The inductance can remain unchanged because the equal height and heat flux design method is used in the optimization process. Thus, the maximum temperature rise becomes the most important parameter which needs to be validated.

Currently, the FEM is widely used to calculate the temperature rise of transformers and reactors, and the accuracy has been verified compared with the experimental results of the prototype [22-26]. As a result, the temperature field simulation results are selected as the evaluation standard for the optimization design results.

According to the design results in Table 2, a fluid-thermal-coupled 2D finite element model is established to calculate the maximum temperature rise of the reactor. Some simplifications and equivalencies are performed: only considering the steady-state thermal process, the influence of the spider arm and sustaining bar are ignored, the reactor is equivalent to the two-dimensional axisymmetric model. The width of the air ducts, and the height and heat load of the encapsulations in the model, are basically identical to the actual design parameters of the reactor.

The boundary conditions of the simulation model are set as follows $[27,28]$ : The radial velocity of fluid in the axis of symmetry is zero, the surface of the encapsulations is a static wall, the radial and axial velocities are zero. In the above boundary of the model, the pressure, fluid velocity, and temperature are defined variables. In the right boundary of the model, the pressure is a defined variable, the velocities of the fluid in all directions are zero, and the temperature is set $20^{\circ} \mathrm{C}$. In the lower boundary of model, the pressure is a defined variable, the velocities of the fluid in all directions are zero, and the temperature is set to $20^{\circ} \mathrm{C}$. The environment temperature is set to $20^{\circ} \mathrm{C}$

Figure 14 shows the temperature field simulation results based on the design results in Method 2, where the maximum temperature rise is $49.5^{\circ} \mathrm{C}$ : Figure 15 shows the temperature field simulation results based on the design results in Method 3, where the maximum temperature rise is $50.1{ }^{\circ} \mathrm{C}$. They only show a $3.1 \%$ and $4.4 \%$ difference compared with the maximum temperature rise of the initial parameters. Meanwhile, the temperature distribution of the inner encapsulation is basically the same. Thus, the simulation results verify the correctness of the comprehensive optimization method. 


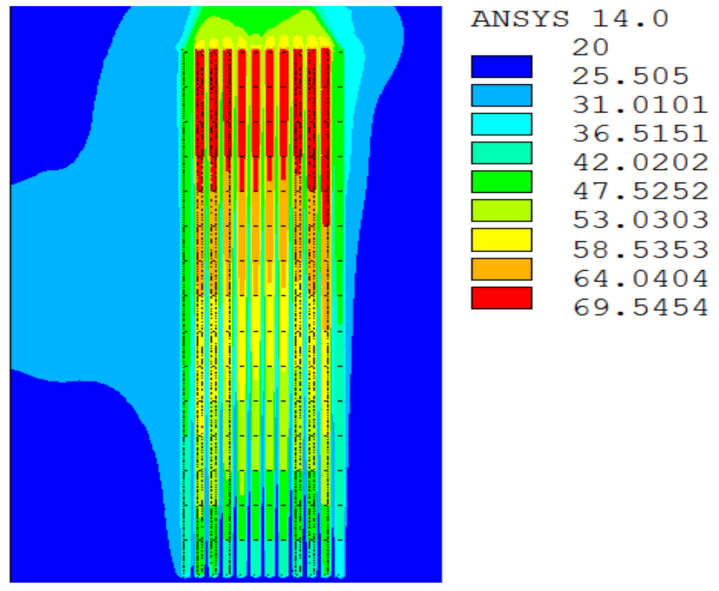

Figure 14. Temperature field simulation results considering the change of air duct width.

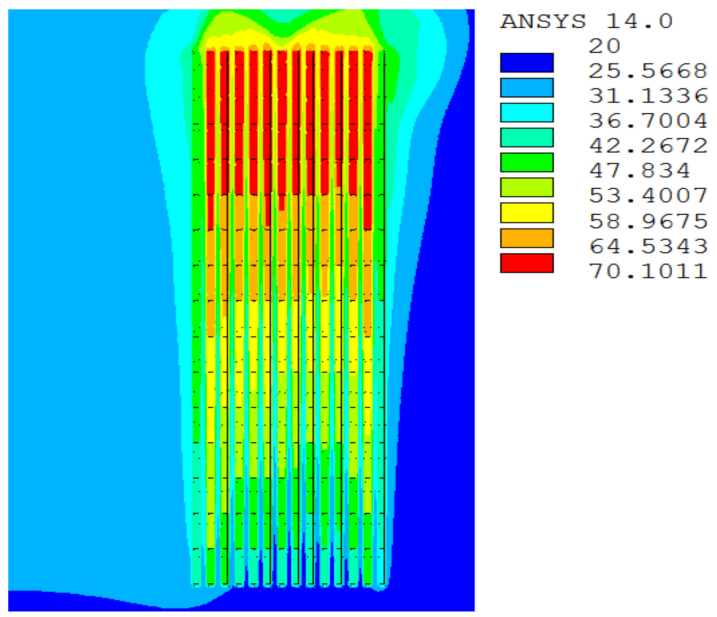

Figure 15. Temperature field simulation results considering the change of air duct width and encapsulation number.

\section{Conclusions}

In this paper, the change of air duct width and encapsulation number are considered in the thermal and electromagnetic optimization of the reactor, in order to realize the minimum metal conductor usage, a comprehensive optimization method is proposed and the correctness is verified by the temperature field simulation results. Meanwhile, the following conclusions can be reached.

The increase of air duct width, encapsulation number, and encapsulation height can obviously improve the thermal efficiency of the encapsulation-air ducts unit, but the electromagnetic efficiency is decreased. In Method 3, the thermal efficiency is improved, but the electromagnetic efficiency is reduced compared with the initial parameters of the reactor, which shows that the comprehensive optimization method adopts a compromise to realize the metal conductor usage minimum.

Compared with the initial parameters of the reactor, the proportionality factor of metal conductor usage is only 0.61 in Method 3, it shows that the comprehensive optimization method can significantly reduce the metal conductor usage, improving the utilization of the metal conductor. Meanwhile, the proportionality factor of the DC resistance becomes 2.38 in Method 3, thus, the comprehensive optimization method leads to the increase of DC resistance.

The thermal efficiency optimization method about encapsulation-air ducts unit can also be potentially applied to the design of an iron core reactor, to improve the thermal efficiency of the coils. 
Acknowledgments: Financial support was provided by the project of Guangzhou Power Supply Co., Ltd. "Research of high capacity short circuit current interruption device and its application" (K-GZM2014-031).

Author Contributions: Fating Yuan and Zhao Yuan completed the main work of this paper, Lixue Chen, Junjia He and Yuan Pan put forward valuable suggestions about the overall structure of this paper, Yong Wang and Junxiang Liu provided the help about the establishment of temperature field simulation model, and all the authors contributed to the discussion, information collection, and the manuscript preparation.

Conflicts of Interest: The authors declare no conflict of interest.

\section{Symbols and Abbreviations}

a The radial width of encapsulation

$b \quad$ The single turn axial height of encapsulation

$C_{p} \quad$ The specific heat at constant pressure

d The width of air ducts

D The diameter of encapsulation

Dav The average diameter of thick wall coils

$H \quad$ The height of thick wall coils

I The current of encapsulation

$k_{a d} \quad$ The ratio value of radial width of encapsulation to air duct width

$k c \quad$ The ratio of total losses to resistance losses

$k x \quad$ The proportionality factor of $x$

$L \quad$ The inductance value of the reactor

$m \quad$ The encapsulation number of the reactor

$\bar{M} \quad$ The mass flow rate of fluid in air ducts

Mass The metal conductor usage of reactor

$\mathrm{Nu} \quad$ The Nusselt number in air ducts

$q_{w} \quad$ The heat flux conducted from encapsulation surface to air ducts

$R \quad$ The resistance of the reactor

$S \quad$ The area of the single turn conductor

$T \quad$ The temperature of encapsulation surface

$T_{\infty} \quad$ The temperature of fluid in air ducts

$\mathrm{TH}$ The thickness of thick wall coils

$T_{\max } \quad$ The maximum temperature rise of encapsulation

$\bar{u} \quad$ The average velocity of fluid

$\bar{W} \quad$ The average turn number

$\rho \quad$ The fluid density of fluid

$\lambda \quad$ The heat conductivity coefficient of fluid

$g \quad$ The acceleration of gravity

$\beta \quad$ The thermal expansion coefficient of fluid

$\rho_{1} \quad$ The mass density of metal conductor

$\mu_{f} \quad$ The dynamic viscosity corresponds to the fluid temperature

The dynamic viscosity corresponds to the encapsulation surface

$\mu_{w} \quad$ temperature

$\gamma \quad$ The conductivity of metal conductor

$v \quad$ The kinematic viscosity of fluid

\section{Appendix A}

According to the average velocity of fluid in air ducts, the Reynolds number can be calculated, as shown in Equation (A1).

$$
\operatorname{Re}=2 \bar{u} d / v
$$

The flow state of fluid in air ducts is decided by Reynolds number, the test results show that the fluid is laminar flow when $R e<2200$, is transition flow when $2200 \leq R e \leq 10^{4}$, and is turbulent flow 
when $R e \geq 10^{4}$. Thus, the experimental correlation of Nusselt number $N u$ can be obtained according to the flow state of fluid.

(1) Laminar flow: The Nusselt number can be written as [18]:

$$
N u=1.86\left(\operatorname{RePr} \frac{2 d}{H}\right)^{\frac{1}{3}}\left(\frac{\mu_{f}}{\mu_{w}}\right)^{0.14}
$$

The Prandtl number Pr in Equation (A2) is defined as:

$$
\operatorname{Pr}=v C_{p} / \lambda
$$

where $\mu_{f}, \mu_{w}$ are the dynamic viscosity which correspond to the fluid temperature and encapsulation surface temperature respectively, and the equation can be used when $\operatorname{Re}<2200, \operatorname{Pr}>0.6$ and $\operatorname{RePr} 2 d / H>10$.

(2) Transition flow: the Nusselt number $N u$ can be written as [18]:

$$
N u=0.0214\left(R e^{0.8}-100\right) \operatorname{Pr}^{0.4}\left[1+\left(\frac{2 d}{H}\right)^{\frac{2}{3}}\right]\left(\frac{T_{\infty}}{T}\right)^{0.45}
$$

where, the equation can be used when $2200 \leq R e \leq 10^{4}, 0.6 \leq \operatorname{Pr} \leq 6.5$ and $0.5 \leq T_{\infty} / T_{w} \leq 1.5$.

(3) Turbulent flow: the Nusselt number $N u$ can be written as [18]:

$$
\mathrm{Nu}=0.023 \operatorname{Re}^{0.8} \mathrm{Pr}^{0.4}
$$

where, the equation can be used when $10^{4} \leq \operatorname{Re} \leq 1.2 \times 10^{5}, 2 d / H \leq 1 / 60$ and $0.7 \leq \operatorname{Pr} \leq 120$.

Meanwhile, the uniform equation can be used for the transition flow and turbulent turbulence in air ducts, as shown in Equation (A6) [20]:

$$
N u=\frac{(f / 8)(R e-1000) P r}{1+12.7 \sqrt{f / 8}\left(\operatorname{Pr}^{2 / 3}-1\right)}\left(1+\left(\frac{2 d}{H}\right)^{2 / 3}\right)\left(\frac{T_{\infty}}{T}\right)^{0.11}
$$

The parameter $f$ in Equation (A6) is defined as:

$$
f=(1.82 \lg R e-1.64)^{-2}
$$

where, the equation can be used when $2300 \leq \operatorname{Re} \leq 10^{6}$ and $0.6 \leq \operatorname{Pr} \leq 10^{5}$.

\section{References}

1. Yu, Z.; Wang, S. Optimum design of dry-type air-core reactor based on coupled multi-physics of reconstructed finite element model. Trans. China Electrotech. Soc. 2015, 30, 71-78.

2. Lin, Q.; Liu, Q.; Liang, Y.; Liu, T.; Li, X. Research of monitoring method for interturn short circuit fault of air-core reactor. Power Capacit. React. Power Compens. 2015, 36, 96-100.

3. Huang, J.; Xiao, Z.; Chen, G. The calculation and design of air core reactor. Electr. Drive 1991, 5, 46-55.

4. Luo, L.; Zhao, S.; Xu, J.; Wei, L.; Lou, Y. Design of Large Inductance Power Cable. High Volt. Eng. 2015, 41, 2635-2642.

5. Li, Y.; Zhang, Z.; Zhu, L.; Jiang, M. Calculation and design of dry-type air-core reactor. Transformer 2013, 50, 1-6. [CrossRef]

6. Li, Y.; Zhang, Z.; Li, L.; Li, G.; Jiang, M. Calculation and design of dry-type air-core reactor. Energy Power Eng. 2013, 5, 1101-1104. [CrossRef]

7. Zhang, C.; Zhao, Y.; Ma, X. Optimization design of separated dry-type air-core reactor based on modified differential evolution algorithm. In Proceedings of the Third International Conference on Information Science and Technology, Yangzhou, China, 23-25 March 2013. 
8. Liu, Z.; Ouyang, S.; Geng, Y.; Wang, J. Study of genetic algorithm in the optimum design of air-core reactor. Adv. Technol. Electr. Eng. Energy 2003, 22, 45-49.

9. Ouyang, S.; Liu, Z.; Geng, Y.; Wang, J. A new improved genetic algorithm and its application in the optimum design of the reactor. Comput. Eng. 2003, 29, 16-17.

10. Zhao, Y.; Chen, F.; Kang, B.; Ma, X. Optimum design of dry-type air-core reactor based on the additional constraints balance and hybrid genetic algorithm. Int. J. Appl. Electromagn. 2010, 33, 279-284.

11. Lu, S.; Sun, C.; Lu, Z. An improved quantum-behaved particle swarm optimization method for short-term combined economic emission hydrothermal scheduling. Energy Convers. Manag. 2010, 51, 561-571. [CrossRef]

12. Smolka, J.; Nowak, A.J. Shape optimization of coils and cooling ducts in dry-type transformers using computational fluid dynamics and genetic algorithm. IEEE Trans. Magn. 2011, 47, 1726-1731. [CrossRef]

13. Yan, X.; Dai, Z.; Yu, C.; Qi, Y. Research on magnetic field and temperature field of air core power reactor. In Proceedings of the International Electrical Machines and Systems (ICEMS2011), Beijing, China, 20-23 August 2011.

14. Yuan, Z.; He, J.; Pan, Y.; Yin, X.; Luo, C.; Chen, S. Research on electromagnetic efficiency optimization in the design of air-core coils. Int. Trans. Electr. Energy Syst. 2015, 25, 789-798. [CrossRef]

15. Yuan, F.; Yuan, Z.; Wang, Y.; Liu, J.; Su, H.; He, J. Research of Electromagnetic and Thermal Optimization Design on Air Core Reactor. IEEJ Trans. Electr. Electron. Eng. 2017.

16. Liu, Q.F.; Dang, H.G.; Liang, Y.C.; Miao, H.T. Optimization algorithm of the reactor design. Power Capacit. React. Power Compens. 2011, 32, 47-60.

17. Chen, F.; Zhao, Y.; Ma, X. Optimum design of dry-type air-core reactor based on design variable reconstruction. Proc. Chin. Soc. Electr. Eng. 2009, 29, 99-106.

18. Wu, T.; Wang, X.; Xu, G.; Pan, Y.; Chen, W. Engineering Heat Transfer; Huazhong University of Science and Technology Press: Wuhan, China, 2011; pp. 120-130.

19. Gnielinski, V. New equations for heat and mass transfer in the turbulent flow in pipes and channels. NASA STI/Recon Tech. Rep. A 1975, 41, 8-16.

20. Gnielinski, V. New equations for heat and mass transfer in turbulent pipe and channel flows. NASA STI/Recon Tech. Rep. A 1976, 16, 359-368.

21. Sun, $X$. The design of air core reactor. Transformer 1988, 11, 7-10.

22. Cao, J.; Cheng, T.; Jiang, Z.; Wen, X.; Zhang, M. Coupling calculation of temperature field for dry-type smoothing reactor. In Proceedings of the International Conference on Electrical Machines \& Systems ICEMS 2014, Hangzhou, China, 22-25 October 2014; pp. 3259-3263.

23. $\mathrm{Wu}, \mathrm{D}$. Temperature field distribution and infrared temperature measurement method research of $35 \mathrm{kV}$ dry-type reactor. Transformer 2013, 50, 62-65.

24. Chen, F.; Zhao, Y.; Ma, X. An efficient calculation for the temperature of dry air-core reactor based on coupled multi-physics model. In Proceedings of the 2012 Sixth International Conference on Electromagnetic Field Problems and Applications, Dalian, China, 19-21 June 2012.

25. Zhang, Y.J.; Qin, W.N.; Liang, G.; Ruan, J.J.; Huang, T. Analysis of temperature rise in reactors using coupled multi-physics simulations. In Proceedings of the IEEE International Conference on Applied Superconductivity \& Electromagnetic Devices, Beijing, China, 25-27 October 2013.

26. Yuan, F.; Yuan, Z.; Liu, J.; Wang, Y.; Mo, W. Research on Temperature Field Simulation of Dry Type Air-Core Reactor. In Proceedings of the 20th International Conference on Electrical Machines and Systems (ICEMS 2017), Sydney, NSW, Australia, 11-14 August 2017.

27. Yuan, F.; Yuan, Z.; Wang, Y.; Liu, J.; Su, H.; He, J. Thermal Optimization for Nature Convection Cooling Performance of Air Core Reactor with the Rain Cover. IEEJ Trans. Electr. Electron. Eng. 2017.

28. Yuan, Z.; He, J.; Pan, Y.; Yin, X.; Ding, C.; Ning, S.; Li, H. Thermal analysis of air-core power reactors. ISRN Mech. Eng. 2013, 2013, 1-6. [CrossRef]

(C) 2017 by the authors. Licensee MDPI, Basel, Switzerland. This article is an open access article distributed under the terms and conditions of the Creative Commons Attribution (CC BY) license (http:/ / creativecommons.org/licenses/by/4.0/). 